\title{
When Experimental study meets Ancient Chinese language study: A Comparative study or Complementary verification study
}

\author{
Yancheng Yang \\ College of Foreign Languages, Hunan University, Changsha, PR China \\ yanchengyang@hnu.edu.cn
}

Keywords: experiment; ancient language; complementary verification; artefacts

\begin{abstract}
Recently experimental study paradigms are widely applied into the language study. The experimental results about the topic "Interactions among space, time and number" can be attested or identified by the linguistic expressions and Symbolic Cognitive Artifacts in Ancient China. To some extent, the language study is a basic complementary verification for experimental study. In this paper, I will use these symbolic cognitive artefacts (the Yellow River Map and the Luoshu Square) in Yi-Ching as a window into the mind, and discuss them in relation to cognitive archeology. And I give a full account of the artefacts, including a full account of the textual sources to understand how these artefacts were employed. Finally I claim that the artifacts can provide a window into the mind, just like language does.
\end{abstract}

\section{Introduction}

Space, time, and number are the three basic dimensions or domains about how we act within and reason about the world, which are systematically intertwined in behavior, language, and the brain. That is to say, not only for experimental study in behavior and the brain, but also for language study, we can't neglect the three domains. Recently, cross-domain interactions study is a popular topic. Interactions between space, time, and number have become a major issue in cognitive science. These interactions suggest that the representation of both time and numbers might be deeply rooted in cortical networks that also benefit spatial cognition[1][2][3][4][5][6]. Especially for Winter (2015), there are mainly two articles talking about cross-domain interactions. One is "Of magnitudes and metaphors: cognitive interactions between space, time and number" [6], the other one is "Mental number space in three dimensions" [7]. Dehaene (2011) in his book Space, Time and Number in the Brain also discussed about the relationship about the three domains[5].

In this paper, I will mainly consider Winter's research[6][7] and Dehaene's book[5] as my references. My general hypothesis is like the following: To some extent, the Neuropsychological experiment results or cognitive neuroscience achievements about the topic "Interactions among space, time and number" can be attested or identified by the linguistic expressions and Symbolic Cognitive Artifacts in Ancient China. Sometimes, if we compare some results in Experimental study with Ancient Chinese language study, maybe we will think about this question-When "the interaction between space, time and number" getting from the Experimental study meets "the interaction between them" from the symbolic cognitive artifact in Ancient Chinese language study, what will happen? In my research I will use the specific linguistic corpus analysis in the two books about Yi-Ching and artefacts interpretation of the Yellow River Map and Luoshu Square in YiChing (One book is in Early Qing dynasty- Hu Wei's book Yi Tu Mingbian, the other one is in Later Ming dynasty-Fang Kongzhao's book The Shi theory in the Book of Changes) [8][9]to explain which one is more appropriate-A Comparative study or Complementary verification study. 


\section{Winter's research(2015) on space, time and number in the brain}

\subsection{ATOM and CMT’s Explanation}

Winter, et al claims that the two theories (ATOM-A theory of magnitude and CMT-cognitive metaphor theory) "shed light on different aspects of the complex interconnections between space, time and number." [6] Interactions between space, time, and number can be well explained by both theories. For ATOM, it mainly has two key behavioral predictions. One is behavioral interactions between space, time and number, such that people should associate "more" in one domain with "more" in another ("more A-more B") [10].The other one is space, time and number should influence action [10]. CMT usually consider an observation about linguistic behavior as its starting point. When we discuss domains of number and time, we will map them to the "source" domain of space. According to CMT, Lakoff (2008) and Lakoff \& Johnson (1980) proposed that conceptual metaphors consist of directional, asymmetric mappings from source (e.g., space) to target domains (e.g., number, time) rather than symmetrical associations between domains[11] [12]. For example, the language of space is re-used for time and number, while the language of time and number are used less frequently to describe space. That is to say, CMT argues that we conceptualize time and number in terms of space. In this way, Winter used the two theories to explain on different aspects of the complex interconnections between space, time and number[6].

Meanwhile, CMT and ATOM agree that "cross-domain influences are most likely bidirectional." [6] Winter claimed that even the linguistic evidence can support bidirectional links between domains[6]. However, there are some Asymmetry (Domain Asymmetry and Directional Asymmetry) among them. At the linguistic level, the language of space is commonly used to describe other domains, time and number in particular [13] [14] [15] [16] [17]. On the other hand, Winter hold that "there are few linguistic connections between numerical and temporal magnitudes" [6].

However, language is one window into cognition. Another may be symbolic cognitive artifacts. "Symbolic cognitive artifacts materially and semiotically mediate human cognition, and are not merely informational repositories, but co-agentively constitutive of culturally and historically emergent cognitive domains"[18]. Calendrical notion is an example of the category of symbolic cognitive artefacts. Before the Zhou dynasty, the ancient Chinese used a solar calendar. We have our ancient calendar at that period, the traditional Chinese calendar is a lunisolar calendar which reckons years, months and days according to astronomical phenomena. There are two original ancient methods to help us know the lunisolar calendar in ancient: One is about an ancient Chinese sundial consisting of an elongated dial and one or two gnomons;The other one is clepsydra or water clock. In this way we can consider the Yellow River Map and the Luoshu Square (the original source of Yi-Ching) as our symbolic cognitive artefacts. For Yi-Ching study, in the language description of symbolic cognitive artifact, linguistic connections between numerical and temporal magnitudes really exist. For example, in the symbolic cognitive artifact of the Luoshu Square, according to Hu Wei (1633-1714)'s book Yi Tu Mingbian and Fang Kongzhao's The Shi theory in the Book of Changes , each black or white circle can represent a number-the odd and even numbers alternate in the Lo Shu pattern; the 4 even numbers are at the four corners (different spatial positions), and the 5 odd numbers (outnumbering the even numbers by one) form a cross in the center of the square. The sums in each of the 3 rows, in each of the 3 columns, and in both diagonals, are all 15 (the number of days in each of the 24 cycles of the Chinese solar year).

As the Yellow River Map's right rotation, we can get the Luoshu Square (Frame of reference, moving time). In this way, one locates in the north, together with six; Two locates in the south, together with seven; Three locates in the east, together with eight; Four locates in the west, together with nine; Five and ten locates in the middle, which are pivot( hub) for heaven and earth's travel.(By Zhangli,, from Huwei’s Yi Tu Mingbian p.96)

The number in Square is generated like in a circle. The middle five adding the north one is six. So the left rotation of northwest is six. The north one adding six is seven, so the west can get seven. 
The west seven adding the southwest two is nine, so the right south can get nine. Nine adding the southeast four is thirteen, divided by ten, three left, so the right east is three. Three adding the northeast eight is eleven, divided by ten, one left, so the right north is one. (By Fang kongzhao, from Fang Kongzhao's The Shi theory in the Book of Changes. vol.1)

According to their interpretation, we can identify that actually there are few linguistic connections (Though we can find there is a background here-moving time) between numerical and temporal magnitudes.

\subsection{Winter's (2015a) evaluating asymmetry}

In Winter's (2015a) article, he clarified and evaluated the issue of asymmetry, and evaluated that "The two theories do not only make different predictions about the direction of the influence between domains (Directional Asymmetry), but also disagree about which domains should have interactions in the first place (Domain Asymmetry).” [6] Specifically speaking, ATOM can correctly predict Directional and Domain Symmetries, while CMT emphasizes the complex chains of reasoning from conceptual metaphors, which may play an important role in higher cognitive processing, and the resulting directionalities and asymmetries.

Winter also mentioned there is still a problem ( has been solved yet), that is "Future empirical work will need to investigate whether there is actually evidence that spatial representations mediate established interactions between number and time.”[6] However, Yi-Ching as a cognitive symbolic artifact can be the empirical work and explain that spatial representations mediate established interactions between number and time. For example, in the Yellow River Map, the ancient people can use the different spatial positions to tell us something about the numeral notion and the temporal practice. The north is in the below, there is a white circle inside, six black circles outside(the distribution of the different circles in number can closely correspond with aspect astrology, then interact with the temporal practice); It can represent the "water" from the view of five elements, which can be related to the temporal practice-before the winter solstice, about November.

There is also linguistic evidence from Fang Kongzhao(1590-1655)'s book The Shi theory in the Book of Changes for it. Paragraph 1, 2 and 3 are quoted from Fang Kongzhao(1590-1655)'s book The Shi theory in the Book of Changes. The following are my translation and interpretation.

One is the beginning of "number", which appears in the north (always connected with Qian trigram, which can represent the beginning and motivation of creating all things in nature). The heaven begins with the $\mathrm{Zi}$ Shi (The heaven is the most important factor in ancient people's mind, then the earth, last human. The earth begins with Chou Shi, human born in Yin Shi, Zi Chou Yin is the first three of earthly branches in Ancient China which can record the time).The Four Shi begins with the Winter Solstice.Then the south relates with Two. One and Two combines into generating Three, which is arranged in the East. In this way, combing One and Three into generating Four, which stays in the West. Combing One and Three into generating Five, which is arranged in the middle. At last, one combining with Five, Six, Seven, Eight, and Nine can generate outside NorthSix, outside South-Seven, outside East-Eight, outside West-Nine, outside Middle-Ten,...

In the Yellow River Map, the Northwest is one and four, Southeast is two and three, the "generating" number (shengshu) is Five. One adds five, forming six, which lies in the North; Two adds five, forming seven, which lies in the South; Three adds five, forming eight, which lies in the East; Four adds five, forming nine, which lies in the West; Five adds five, forming ten, which lies

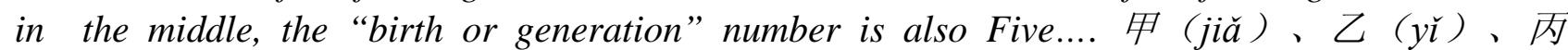

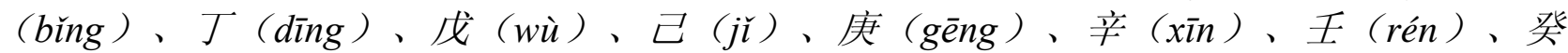
(guí) are Ten Chinese Heavenly Stems. Inside circle of the map is Jia (The first of the Heavenly Stems),Bing-third ,Wu-fifth, Geng-seventh , and Ren-ninth, they belong to Yang ; Outside circle of the map is Yi-second, Ding-fourth, Ji-sixth, Xin-eighth, and Gui-tenth, they belong to Yin. The five elements-wuxing depends on number "ten", and then it interacts with "space"-five positions and "time"-four seasons. 
The interpretation of the Yellow River Map has to do with the "five elements"(wuxing) and the five cardinal directions. The correspondence between five elements, positions, numeral dots and seasons is like the following:

Water-One white dot inside, six black dots outside-North-November,Winter

Wood- Three white dots inside, eight black dots- outside-East-March,Spring

Fire- Seven white dots outside, two black dots inside-South-July,Summer

Metal- Nine white dots outside, four black dots inside-West-September,Autumn

Earth-Five white dots inside, ten black dots outside-Middle-May

What we find in Yi-Ching is not the three separate domains, space, time and number, but perhaps a single domain, ancient people perhaps conceptualize each domain through the other domains. In particular, we can find some language evidences in the two books to show how is number represented in the spatial array independently of the NUMBER of dots and how is this related to time?

As for the reason why in ancient people did this kind of correspondence, especially about the relationship between spatial position, numeral expression, and temporal practice in the Map, the "generative" cycle and the "destructive" or "conquering" cycle of "wuxing" can help our understanding .

From the above linguistic analysis of Yi-Ching, we can find that there are some interactions between spatial cognition, numerical cognition and temporal cognition, that is also the Ancient Chinese's way to show us the rules in the world. What is more, Winter (2015a) pointed "A large number of experimental findings from neuroscience and experimental psychology demonstrated interactions between spatial cognition and numerical cognition.” [6]

There is also various empirical support widely across levels for Directional Asymmetry. "Directional Asymmetry at the perceptual or conceptual levels is less reliable than asymmetries at the linguistic level but nevertheless attested. Where might they come from?" [6] Meanwhile, there is a question proposed by Winter (2015a)-“How many languages use spatial terms to describe number and time?” From the study of Yi-Ching, Ancient Chinese is actually one kind of language which usually use spatial representation terms (eight trigrams, bagua-three changing lines, the hexagram, yao-six changing lines ,etc in Yi-Ching) to describe number and time. Taking the six lines of Qian hexagram for example, from below to above, each changing line represent one of the temporal and numeral concept, the first below line-Beginning 9 (初九 -one situation or condition)represent the beginning period of an event or thing, which means something is not obvious, just has signs or auspice(sometimes omen); The second below line- Second 9 (九二) represent the primary development period of an event or thing, which means something is obvious and has some development;...The sixth line(above one )- Top 9 (上九) represent the end period of an event or thing, which means something is finished or ended and will have another cycle.It seems that there is a kind of time interval concept here. Actually, each line refers to the spatial notion, which can combine with number to represent the different period of temporal concept. To some extent, this kind of spatial temporal and numeral concept and representation is periodic and opportunistic, even cyclic (Things will develop in the opposite direction when they become extreme.).

From Winter's view, we get the point that Magnitudes is connected with ATOM; while relations between conceptual elements is closely connected with CMT. Their difference is often overlooked. If we want to know the distinction between relations and magnitudes, usually we will think about the question whether ATOM can account for associations between spatial locations and number or time [19] [20] [21]. There is also various empirical support widely across levels for Directional Asymmetry. "Directional Asymmetry at the perceptual or conceptual levels is less reliable than asymmetries at the linguistic level but nevertheless attested. Where might they come from?” [6] 


\section{Dehaene's study (2011) on Space, Time, and Number in the brain}

Dehaene (2011) showed us that the study of mathematical cognition and the ways in which the ideas of space, time and number are encoded in brain has become a fundamental issue for neuroscience. [5]

What do the representations of space, time and number have in common? Do the representations of space, time and number share neural resources? Or do they interact through systematic crossdimensional mappings? Or is it a single dimension, for instance space, used as a reference for all the others, as is perhaps suggested by the observation that, in human languages, spatial terms are frequently used metaphorically to refer to time and number? This book dealt with these questions. Well, there is some evidence of a degree of innateness in humans and some animals that space, time and number are part of the essential cognitive tools[5].The representations of space, time and number all raise deep computational issues for cognitive neuroscience; Indeed, our understanding of the external world really need these basic concepts, we find it hard to imagine "how any animal species could survive without possessing mechanisms for spatial navigation, temporal orienting (e.g.,time-stamped memories), and elementary numerical computations (e.g., choosing the food patch with the largest expected return)" [5]; The papers in the book proved that the representations of space, time and number share neural resources, such as the nervous system; They interact through systematic cross-dimensional mappings, and space can be used as a reference for all the others, as is perhaps suggested by the observation that, in human languages, spatial terms are frequently used metaphorically to refer to time and number.

However, in both Winter (2015) and Dehaene (2011)'s studies, the common premise is that they all consider time, space and number as three domains. And all their studies belong to the area of neuroscience, cognitive psychology, developmental science, neuroimaging, neuropsychology and theoretical biology, they are modern science. How about Ancient Chinese language study about it? I mean whether Ancient Chinese language can represent people's conceptualization of time, space and number in that period. If we can find the relationship among the three notions through Ancient Chinese language or cognitive symbolic artefacts, even though it may be different from the experimental study, it can also be a complementary reference. What is more, none of the Chinese Yi-Ching Scholars focus on the study about "Cognitive Interactions among space, time, and number”.

\section{Ancient Chinese language study is a complementary verification study for Experimental study}

In our organizing Cognitive science, we attempt to think of each of space, time and number as a separate domain, maybe because we have a discipline called Physics, which consider space and time as separate, in Physics space has 3 dimensions, time has 1 dimension, and they have variables, etc. That is to say, only in classical Newtonian physics, not in Einstein's relativistic physics, space and time are separate domains. And then we have another discipline called Mathematics, which is about the theory of number. However, in Ancient China, especially about the original Yi-Ching period(the early historical times in ancient-shanggu period, for example, Fuxi period, Fuxi eight hexagram is the oldest version, etc), we do not have a real discipline called Mathematics, even no discipline called Physics. But at that period Ancient people have their special way of thinking about space and time, the original idea of "shi" and "wei" in Yi-Ching can prove. General speaking, each of 64 hexagrams can represent one special "shi”, every hexagram includes six yaos (lines), which can represent six different " wei” happening in the same "shi”. What is more, "shi” has two kinds of representations, one is about the description about time flowing, the changing of four seasons and so on (nature), the other one is about human's activity, fate, etc (event based). "wei” also includes two kinds, one is about permutation and combination of semiotic position like "-- broken line" and “-whole line”, the other one is about human's living situation, social status, circumstance, etc. The 
former “wei” is like “初、二、三、四、五、上”, the later “wei” is always connected with “junzi 君子”, “xiaoren小人”, “daren大人”etc.

Strictly speaking, we have our original discipline Mathematics and Physics in ancient, though to some extent they are a little different from the modern western discipline. However, the time when Mathematics and Physics appears in ancient is later than the appearance of the original YiChing (Shanggu period). We can find a lot of classical ancient works to show our evidences. For example, The Zhoubi Suanjing, or Chou Pei Suan Ching(周髀算经), is one of the oldest Chinese mathematical texts. "Bi" means thigh and according to the book, it refers to the gnomon of the sundial. The book is dedicated to astronomical observation and calculation. "Suan Jing" or "classic of arithmetic" were appended in later time to honor the achievement of the book in mathematics; The Mohist canon of the Mo Jing described various aspects of many fields associated with physical science, and provided a small wealth of information on mathematics as well.

In Ancient China, there is a particular cognition of space, time and number. Especially in YiChing, when Ancient Chinese are thinking about things in time, they are also thinking about things in space (always related with number in some aspect); When they are thinking about space, they are also thinking about things in number (always related with time in some aspect); When they are thinking about things numerically, they are also thinking about the way in which they are organizing in distribute (inseparable concepts) space and time. In this way, what we find in YiChing is not the three separate domains, space, time and number, but actually a single domain, they perhaps conceptualize each domain through the other domains. For Yi-Ching, spatial representation of numbers is the main original reference for our cognition of space, time and number. However, Winter (2015b, p.209) also discussed the spatial representation of numbers and claimed that the interactions in three dimensions (Horizontal spatial-numerical associations, Vertical spatialnumerical associations, Distance-based or "sagittal” SNARC effects) between spatial cognition and numerical cognition has been demonstrated lots of past experimental findings from neuroscience and experimental psychology. Here I will show the symbolic cognitive artifact in Yi-Ching(Map and Square) to explain the relationship among the three notions linguistically and conceptually, trying to do complementary verification study with experimental study.

When Fang Kongzhao talked about Kun hexagram in his book -The Shi theory in the Book of Changes, he used the language description like "For the Yellow River Map, in the bottom it corresponds with the Kun hexagram, and is related to number "one(six)", which is in the north; while for Luoshu Square, the Kun hexagram related to number "two", which is in the southwest,..."About Dui hexagram in his book, he said "For the Yellow River Map, on the right it corresponds with the Dui hexagram and is related to number " four and nine", which is in the West and belongs to Jin (one of the five elements),...”[8]

From the language description, we can find that the ancient ancestors always combined the concept of "space" and the concept of "number" (based on the eight diagrams) to acquire the concept of "time". On the linguistic, conceptual and cultural level, their understanding of time is first based on the eight diagrams, then related to the interaction of space and number, just like the following Fig. As Winter (2015a) mentioned, always the evidence for Directional Asymmetry is mixed[6], but the factors at the linguistic level is the strongest evidence for this kind of asymmetry. In this way, I use a full account of the textual sources to understand how the artefacts were employed, I find space is always the source domain for the conceptualization of the target domains of time and number, it is a Complementary verification study for experimental study.

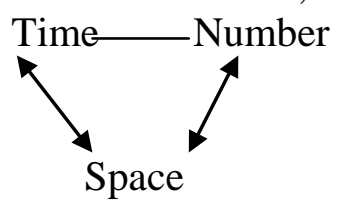

Fig 


\section{Concluding remarks}

To some extent, language can represent something in human's brain. Not only for modern people, but also for Ancient people, they have something common in their brain structure, only for the limitation of technology, the ancient people cannot know neuropsychological evidences about their brain. The language can express or present thought.In ancient people's mind, the certain interaction really exist like the present neuroscience and psychological evidence), which can help us explore into temporal, spatial and numeral concept and representation in ancient china. So I claim that the artifacts can provide a window into the mind, just like language does.

The former studies mostly focus on mental and internal representation, my focus here is mainly about linguistic representation (text analysis and language expression) and other semiotic representations (Symbolic Cognitive Artifacts, iconic maps and squares, etc). Through the specific linguistic corpus analysis in the two books about Yi-Ching and those artifacts interpretation of the Yellow River Map and Luoshu Square in Yi-Ching, I try to answer this question-When Experimental study meets Ancient Chinese language study, what will happen? My finding is that Experimental study is really complementary verification study for Ancient Chinese language study in different aspects. The research paradigm and perspective between them is different, we can't simply compare.

\section{Acknowledgments}

In this paper, the research was sponsored by the Education Ministry Social Science Foundation of China, (Project No. 17YJCZH221) .The author thanks Chris Sinha, and Dr.Winter, Dehaene,etc.

\section{References}

[1] Hubbard, E. M., Piazza, M., Pinel, P., \& Dehaene, S. Interactions between number and space in parietal cortex. Nature Reviews [J], 2005(6), 435-448.

[2] Umiltà, C., Priftis, K., and Zorzi, M. The spatial representation of numbers: evidence from neglect and pseudoneglect. Experimental Brain Research [J], 2009 192(3), 561-569

[3] Walsh, V. (2003). A theory of magnitude: common cortical metrics of time, space and quantity. Trends in Cognitive Sciences [J], 2003(7),483-488.

[4] Dehaene, S., \& Brannon, E. M. Space, time, and number: a kantian research program. Trends in Cognitive Sciences [J], 2010 14(12), 517-519.

[5] Dehaene, S. Space, Time and Number in the Brain[M]. Elsevier Academic Press,2011.

[6] Winter,B., Marghetis, T., \& Matlock, T. Of magnitudes and metaphors:explaining cognitive interactions between space, time, and number. Cortex [J], 2015a (64), 209-224.

[7] Winter, B., \& Matlock, T., Shaki, S., \& Fischer, M. Mental number space in three dimensions. Neuroscience and Biobehavioral Reviews [J], 2015b (57), 209-219.

[8] (Ming) Fang Kongzhao. The Shi theory in the Book of Changes [M].Shanghai: Shanghai Ancient Books Press,2002.

[9] (Qing)Huwei. Proofread by Zheng Wangeng,Yi Tu Mingbian [M]. Beijing: China Publishing House Press,2008.

[10] Bueti, D., \& Walsh, V. The parietal cortex and the representation of time, space, number and other magnitudes. Philosophical Transactions of the Royal Society of London [J], 2009 364(1525), 1831-1840. 
[11] Lakoff, G. The neural theory of metaphor. In R. W. Gibbs(Ed.), Cambridge handbook of metaphor and thought [M].Cambridge, MA: Cambridge University Press,2008.

[12] Lakoff, G., \& Núñez, R. Where mathematics comes from: How the embodied mind brings mathematics into being [M]. New York: Basic Books,2000.

[13] Alverson, H. Semantics and experience: Universal metaphors of time in English, Mandarin, Hindi, and Sesotho[M].Baltimore:Johns Hopkins University Press,1994.

[14] Haspelmath, M. From space to time: Temporal adverbials in the world's languages[M]. Munich, Germany: Lincom,1997.

[15] Lakoff, G., \& Johnson, M.Metaphors we live by[M]. Chicago: University of Chicago Press, 1980.

[16] Sweetser, E. From etymology to pragmatics: Metaphorical and cultural aspects of semantic structure[M]. Cambridge: Cambridge University Press,1991.

[17] Traugott, E. On the expression of spatio-temporal relations in language. In J. H. Greenberg, C. A. Ferguson, \& E. A. Moravcsik (Eds.), Universals of human language [M]. Stanford: Stanford University Press,1978.

[18] Sinha,C.(2015).Language and other artifacts:socio-cultural dynamics of niche construction. Frontiers in Psychology[J], 2015(6), 1-18.

[19] Dehaene, S., Bossini, S., \& Giraux, P. The mental representation of parity and number magnitude. Journal of Experimental Psychology General[J], 1993 122(3), 371-396.

[20] Vicario, C. M., Pecoraro, P., Turriziani, P., Koch, G., \& Caltagirone, C. Relativistic compression and expansion of experiential time in the left and right space. PLoS One[J], 2008 3 (3),1-4.

[21] Winter, B., Perlman, M., \& Matlock, T. Using space to talk and gesture about numbers: evidence from the TV News archive. Gesture[J], 2014(13), 377-408. 